\title{
Hydrogenation and Dehalogenation under Aqueous Conditions with an Amphiphilic Polymer-Supported Nanopalladium Catalyst
}

\author{
Ryu Nakao, Hakjune Rhee, ${ }^{\dagger}$ Yasuhiro Uozumi* \\ Institute for Molecular Science (IMS), Myodaiji, Okazaki 444-8787, Japan \\ uo@ims.ac.jp
}

Received Date (will be automatically inserted after manuscript is accepted)

\section{Supporting Information}

General: ARP-Pd was prepared according to the procedure previously reported by the authors. All starting materials and authentic samples of the products were purchased from Aldrich Chemical Co. Inc. and Tokyo Kasei Co. Ltd., and used without further purification. All catalytic reactions were carried out with shaking on a wrist-action shaker (Burrel Scientific Inc.) or a Peti-Syzer (Hi-Pep Co. Ltd.). GC-MS analysis was performed on a Hewlett Packard HP 6890 with a 5973 Network Mass Detector. All reaction products were identified by comparison with their GC-MS spectra versus authentic data.

General Procedure for Hydrogenation: A typical procedure for the reaction of trans-cinnamyl alcohol in the presence of ARP-Pd in water to give 3-phenyl-1-propanol (Table 1, run 7) is as follows: A mixture of transcinnamyl alcohol $(0.5 \mathrm{mmol})$, ARP-Pd $(65 \mathrm{mg}, 26 \mu \mathrm{mol}$ of $\mathrm{Pd}$ ) in $1.0 \mathrm{~mL}$ of $\mathrm{H}_{2} \mathrm{O}$ was shaken under a hydrogen atmosphere $(1 \mathrm{~atm})$ at $25{ }^{\circ} \mathrm{C}$ for $24 \mathrm{~h}$. The reaction mixture was filtered and the resin beads were rinsed with ether (4 times x $5 \mathrm{~mL}$ ). The combined ethereal washings were dried over $\mathrm{Na}_{2} \mathrm{SO}_{4}$ (100\% conversion; >99\% GC yield). The crude product was chromatographed on silica gel to give $81 \%$ isolated yield of 3-phenyl-1-propanol. The GC-MS spectrum of the product showed good similarity $(>95 \%)$ with the authentic data.

General Procedure for Hydrodechlorination: A typical procedure for the reaction of methyl 4chlorobenzoate in the presence of ARP-Pd to give benzoic acid is as follows: To a mixture of ARP-Pd (60 mg, 24 $\mu \mathrm{mol} \mathrm{Pd})$ and methyl 4-chlorobenzoate $(85 \mathrm{mg}, 0.5 \mathrm{mmol})$ in $10 \%$ aqueous isopropanol $(1.0 \mathrm{~mL})$ was added an aqueous solution of ammonium formate $(63 \mathrm{mg}, 1.0 \mathrm{mmol}$ in $0.2 \mathrm{~mL}$ of $\mathrm{H}_{2} \mathrm{O}$ ) at $25{ }^{\circ} \mathrm{C}$. The mixture was stirred at $25{ }^{\circ} \mathrm{C}$ for $120 \mathrm{~min}$. The reaction mixture was filtered and the catalyst beads were extracted with ethyl acetate $(5 \mathrm{~mL}, 3$ times). The combined extract was washed with brine and dried over $\mathrm{Na}_{2} \mathrm{SO}_{4}$ (100\% conversion; >99\% GC yield). The crude product was chromatographed on silica gel to give $94 \%$ isolated yield of methyl benzoate. The GC-MS spectrum of the product showed good similarity (>95\%) with the authentic data.

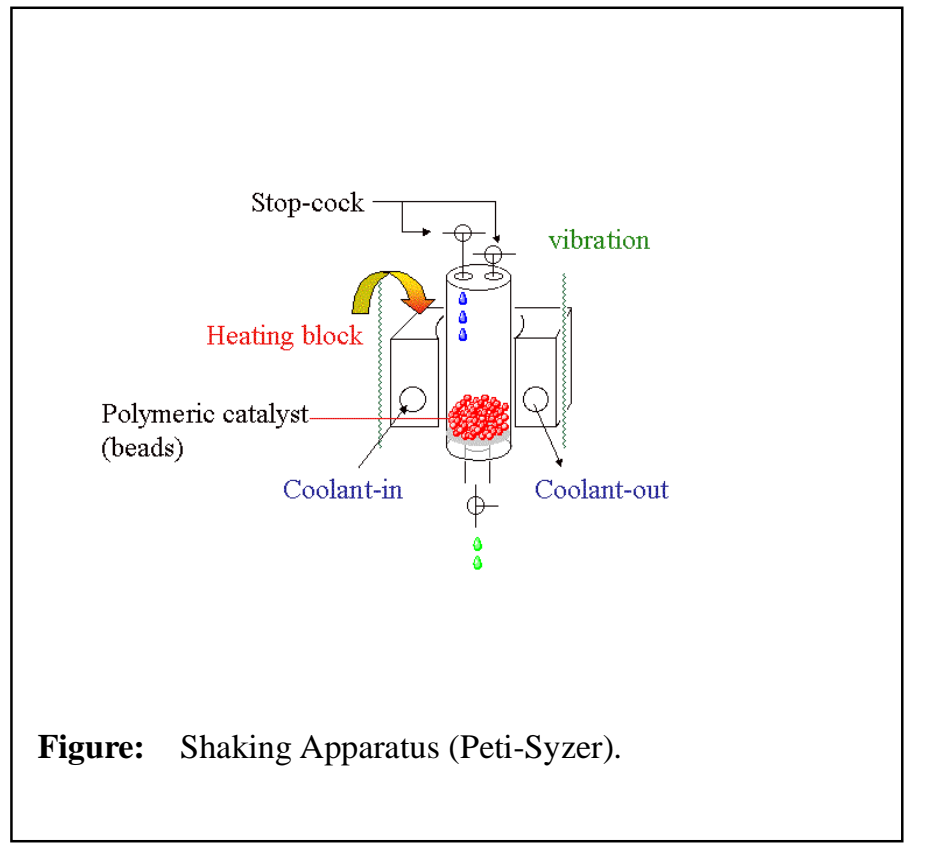

